\title{
The Design of Software Programming for Taxi Price Calculation
}

\author{
Xiaoli Song \\ Jiangxi Modern Polytechnic College
}

Keywords: FPGA; Taxi meter; VHDL; Computer applications

\begin{abstract}
In recent years, integrated circuit and its design and manufacturing tools have developed very rapidly. The appearance of Complex Programmable Logic Device and Field-Programmable Gate Array brought about great changes in electronic design technology. Because of short development cycles, low cost, high reliability, freedom to modify logic functions, FPGA programming are favored by engineers of Electronic Design Automation , and the demand for electronic product is also increasing. Current taxi meter with single-chip microcomputer system has poor stability, inflexible pricing mode easiness to be cracked and cheating, so designing a multifunctional taxi meter based on new technologies has practical and economic value. This paper introduced FPGA technology and carried on a series of improvements on the existing taximeter software and hardware based on VHDL language, and also developed some functions according to industrial applications and geographical characteristics.
\end{abstract}

\section{Introduction}

In the course of the comprehensive development of China's economy, the big cities and the taxi industry also developed rapidly, and taxis have become popular means of transport in people's daily life. The taxi meter is a intelligent instrument of taxi fees and important equipment for the taxi market standardization and reducing disputes between passengers and drivers. The taxi industry is developing very quickly, so users have increasingly higher requirements for taxi meter. Users 1 require the meter to have functions of a stable performance, accurate pricing and features such as deterring cheating; Also requires functions of pricing standard flexibility in its setting, operating sales statistics, password setting. At present, the taxi industry are faced up with high fuel prices and the costs adjustment problems of summer air conditioning, so modifying pricing standard is imperative as oil price rises, and the flexible setting of valuation standards of taxi mete has become an integral part. Traditional taxi multifunctional meter based on single chip design has been far behind the this change. The monolithic integrated circuit is not universal and different chips have different sets of instructions, which caused difficulties of design and development, and feature upgrades are cumbersome and require a restructuring of its hardware and software updates synchronization. It also has high cost, and may occur new destabilizing factors each time. This paper introduced FPGA technology and carried on a series of improvements on the existing taximeter software and hardware based on VHDL language, and also developed some functions according to industrial applications and geographical characteristics.

\section{The Design Methods of VHDL Language}

In VHDL system design, the method of design top-up, that is to say, firstly to introduce and distinguish the whole. On the basis of this, improve the specific features of each module, allow each module's design is reasonable. After the completion of each module, it requires debugging connection to the circuit. So you can design a complete circuit flow as shown in chart 1 . 


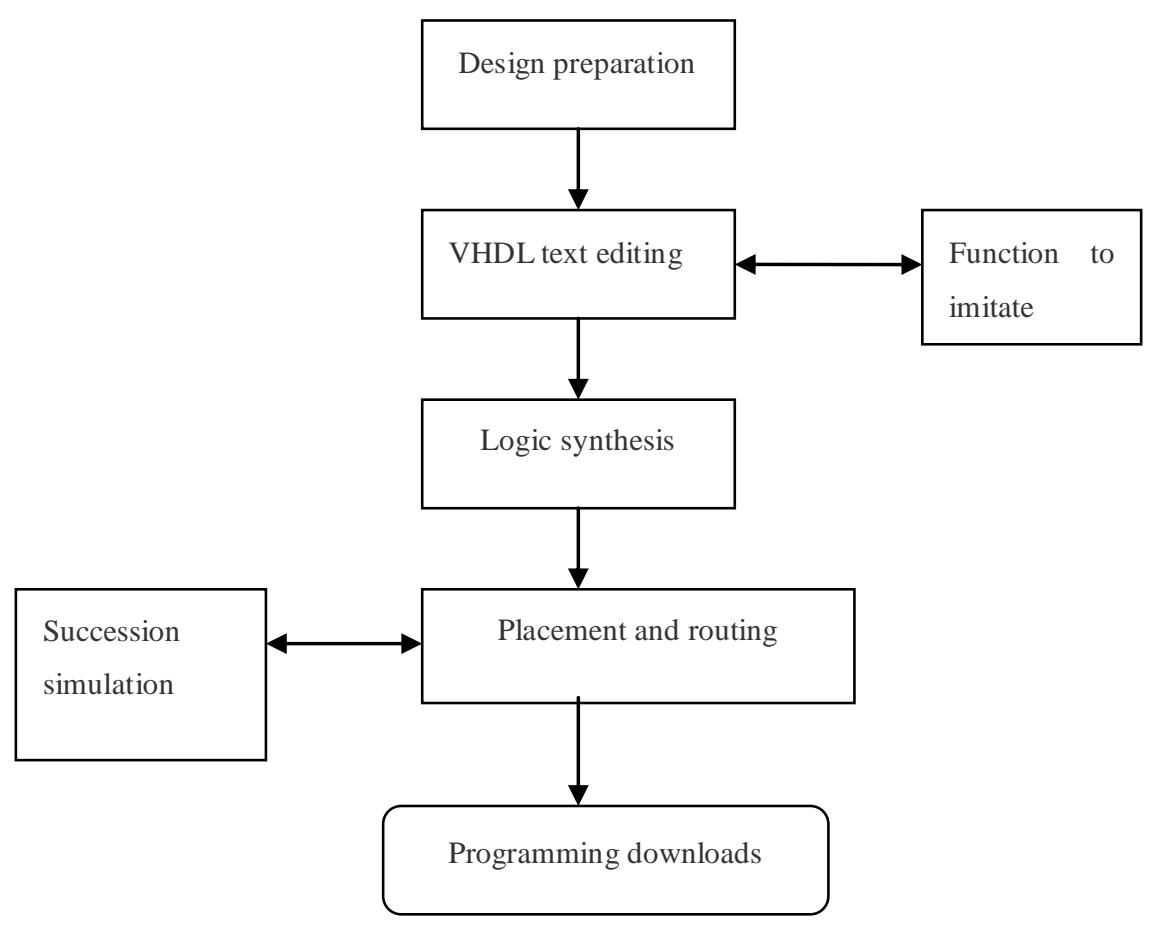

Figure 1. The design processes of VHDL

The development of EDA makes FPGA hardware language design become a trend.i VHDL language complete processes using Quartus II software development of FPGA as follows:

(1)Text editing: Any text editor can be used, you can also use special VHDL editing environment. Usually VHDL files are saved as. vhd files.

(2)Function to imitate: transfer Files into VHDL simulation software and inspect whether logic function is correct (It is also called forward simulation. For the simple design, you can skip this step, we can carry on timing simulation after the wiring is completed)

(3)Logic synthesis: Put the source files into logic synthesis software to synthesize, that is synthesizing language into connection between minimalist Boolean expression and signal. Logic synthesis software generated. EDF (EDIF) the EDA industry standard files

(4)Placement and routing: Put edf files into PLD software provided by the manufacturer for wiring, that is putting the designed logical into PLD/FPGA

(5)Succession simulation: need to use the precise parameters got from Placement and routing and use simulation software to verify the circuit's timing (also called Latter simulation).

(6)Programming downloads: After confirming the simulation, download the file to the chip.SOF files are directly used to download into chips (power will be gone). POF file is used for download mm chip (when power off, it does not go away).

\section{The Design Specifications of Taxi Pricing System}

This design is designed by knowing taxi fare in a city. Because the taxi price is not the same everywhere, the following display the pricing rules of taxi, As shown in Table 1

The above price standard is the ordinary standard, but profession influence can bring about certain change of price, therefore in the aspect of price, we can the nimble control and carry on the real-time adjustment.

The introduction of System process: Taxi meter is consist of vehicle type, speed adjustment module module, the meter module, timing modules, costs the standard modules of set, air-conditioning costs, fuel surcharges, automatic charging module, statistics module, the module 
clock module, the LED display module and other modules. The entire system uses a modular design, firstly writ function of every module with VHDL, and then connect every module together into the pricing system by using the top-level schematic diagram.

Table 1 a taxi charging standard

\begin{tabular}{|c|c|c|}
\hline & Daytime 6: 00-23:00 & The night 23:00-6:00 \\
\hline \multirow{5}{*}{ Cost standard } & Starting $6(3 \mathrm{~km})$ & Starting $8(3 \mathrm{~km})$ \\
\hline & 2/km (10km plus $50 \%)$ & $2.5 / \mathrm{km}(10 \mathrm{~km}$ plus $50 \%)$ \\
\hline & $\begin{array}{c}3 \text { Yuan } / \mathrm{km} \text { ( surpasses the } 10 \mathrm{~km} \text { Canada } \\
\text { to receive } 50 \% \text { ) }\end{array}$ & $\begin{array}{c}4 \text { Yuan } / \mathrm{km} \text { ( surpasses the } \\
10 \mathrm{~km} \text { Canada to receive } 50 \% \text { ) }\end{array}$ \\
\hline & \multicolumn{2}{|c|}{ Every 5 minutes to $1 \mathrm{~km}$ (when the vehicle is lower than the $1 . \mathrm{Okm} / \mathrm{h}$} \\
\hline & \multicolumn{2}{|c|}{ Air conditioning costs, fuel surcharges } \\
\hline
\end{tabular}

\section{System Software Design}

Software Outline. The software system of taxi meter based on single chip microcomputer consists of a main program and several modules of the program. The total flow chart as Fig. 2, and its function is turned on after the Administration and calling a subroutine.

The program of the system consists of several modules including: (1) system monitoring program, (2) time subroutine (3) modified procedure (4) using software logo, and measurement process.

System Monitor Program. System Monitor program is a procedure which controlling single-chip microcomputer system to operate according to the intended operation mode .It completes Man-machine conversation and remote control, making the system complete the specified operation by our intention or remote control commands. It is a framework of single-chip computer system program .Generally speaking, monitor tasks include: system self-check, initialization, keyboard commands, interface command to deal with conditions triggering and display. But in custom, monitoring programs are Keyboard analysis procedure, and other tasks are scattered in the particular modules. In this system we use key code analysis-job scheduling according to design requirements. Because in this design our pressed key is one multi-purpose, as for a-keys-type structure, monitoring procedures can't decide which job is put into operation in accordance a key code obtained already, and it must start a job according to a series of keystrokes. Thus, the same key has different meanings in different series. To this end, we must introduce the concept of System State, namely divide operation system into several States, making each button have only one definition in any state. Thus we use query mode and the timer interrupt mode in Fig. 3:

Fixed time inquiry is mainly used for scanning function key programs, $0 \mathrm{~ms}$ timer interrupt of the clock program 1, and judgment of the automobile running status. It is mainly used for scanning function key programs, $0 \mathrm{~ms}$ timer interrupt of the clock program 1 , and judgment of the automobile running status

\section{Conclusion}

This paper described the principle of meter and timing in detail and made error analysis. FPGA is widely used in communication, control, data, calculation, using FPGA to design products can reduce the risk of electronic systems and development costs; Reduce the market time; reduces the maintenance promotion cost through technologies of system programming and long-distance online restructuring. Using it to realize multi-functional taxi meter based on VHDL language system can save you a lot of peripheral circuits, and be stable, simple and effective, you can design more powerful functions to improve product competitiveness. This paper not only makes the meter have 
surcharge function, but easy to change the pricing parameters, and can also store operational information, providing management with the primary basis to develop relevant policies. The mete designed according to this method has small errors, and its software design is highly flexible.

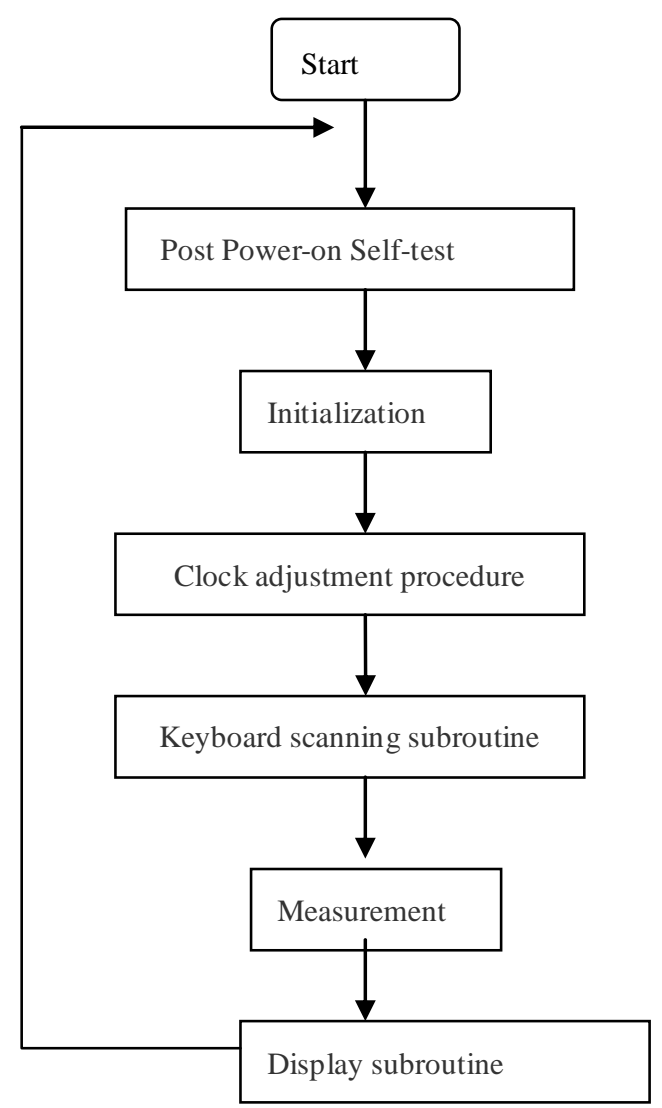

Figure 2. The main flow chart of program

\section{References}

[1] $\mathrm{Ju} \mathrm{H}$. Research and application of short-range communication technology in taxi pricing management system [J]. Microcomputer \& Its Applications, 2011.

[2] Chen L. The Design and Realization of the Information Service System for Taxi Business Based on GPS/GIS[C]// International Conference on Logistics Engineering and Intelligent Transportation Systems. 2010:1-4.

[3] Sun X E. Software Design for Taxi Computation System [J]. Shanxi Electronic Technology, 2007.

[4] Qiu L F. Design of Intelligent Taxation-control Price Calculator in Taxicab [J]. Instrumentation Technology, 2007.

[5] Hosni H E, Farhat N, Nimer R, et al. An optimization-based approach for passenger to shared taxi allocation[C]// International Conference on Software, Telecommunications and Computer Networks. IEEE, 2012:1 - 7.

[6] Cheng V H L, Sweriduk G D. Trajectory design for aircraft taxi automation to benefit trajectory-based operations $[\mathrm{C}] / /$ Asian Control Conference, 2009. Ascc 2009. IEEE, 2009:99-104.

[7] Sui P, Wo T, Wen Z, et al. Privacy Risks in Publication of Taxi GPS Data[C]// IEEE International Conference on High PERFORMANCE Computing and Communications. 2014:1189 - 1196. 
[8] Borgida A, Mylopoulos J, Schmidt J W. The TaxisDL Software Description Language [M]// Database Application Engineering with DAIDA. Springer Berlin Heidelberg, 1993:65-84.

[9] Wei D, Yuan C, Liu H, et al. The Impact of Service Refusal to the Supply-Demand Equilibrium in the Taxicab Market [J]. Networks \& Spatial Economics, 2016:1-29.

[10]Wongpiromsarn T, Xiao N, You K, et al. Road Pricing for Spreading Peak Travel: Modeling and Design [J]. Computer Science, 2012.

[11]Harris W. System and method for vehicle delivery tracking service [J]. 2015.

[12]Morgan E L. Open source software and libraries: A current SWOT analysis [J]. 2010.

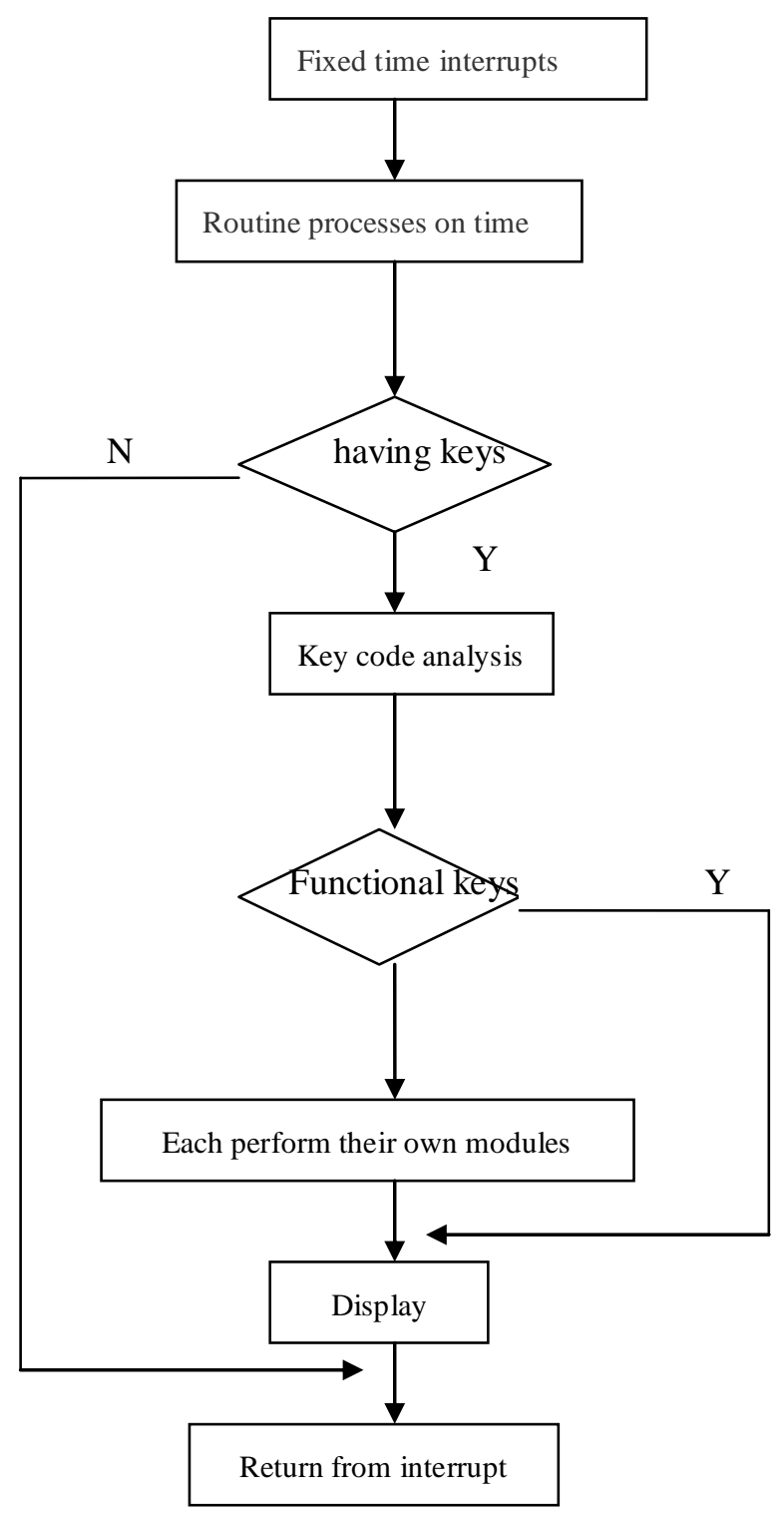

Figure 3. The mode of fixed time interrupts 